\title{
BACTERIOLOGICAL FEATURES OF INFECTIOUS SPONDYLODISCITIS AT MOHAMMED V MILITARY TEACHING HOSPITAL OF RABAT
}

\author{
Zohoun $A^{1^{*}}$, Ngoh Akwa $E^{1}$, El Ochi $M^{2}$, Oragwu $N^{1}$, Akhaddar $A^{3}$, Albouzidi $A^{2}$, El Ouennass $M^{1}$ \\ ${ }^{1}$ Department of Microbiology, Military Teaching Hospital Mohammed V Rabat; ${ }^{2}$ Department of Anatomopathology, Military \\ Teaching Hospital Mohammed V Rabat; ${ }^{3}$ Department of Neurosurgery, Military Teaching Hospital Mohammed V Rabat.
}

Submitted: July 17, 2011; Approved: June 07, 2012.

\begin{abstract}
To review the bacteriological features of infectious spondylodiscitis and provide recommendations for the initial therapy which remains empirical in our context. Retrospective study including patients diagnosed with spondylodiscitis over a period of 4 years (2006-2009) at the Rabat Military Teaching Hospital. During the study period, we analysed 30 cases: the mean age was 49.9 years and 21 cases (70\%) were male. The patients were predominantly hospitalized in neurosurgery department (15/30) followed by rheumatology department (10/30). The site of infection was lumbar in 21 cases (21/30), dorsal in 7 cases (7/30). 26 cultures were positive of which 19 (19/26) were monomicrobial. Tuberculosis (TB) was implicated in 10 cases (10/30) including 4 cases in association with common organisms (Propionibacterium acnes, Staphylococcus aureus, Staphylococcus epidermidis, Corynebacterium species). Brucella melitensis was isolated in 1 case. Infections caused by pyogenic bacteria were isolated in 15 cases of which $12(12 / 15)$ revealed simple organisms including Gram-positive cocci in 9 cases (9/12) with 3 cases of $S$. aureus and Gram-negative bacilli in 3 cases (3/12) with 2 cases of P. aeruginosa. Blood cultures carried out for 16 patients were positive in 7 cases. The anatomopathologic exams carried out for 20 patients found in 6 cases epithelioid granulomata and giants cells with caseous necrosis in total concordance with TB culture. TB is the most frequent cause of spondylodiscitis in Morocco. Our study found the same frequency for nonspecific and specific germs. Empirical treatment must take into account S. aureus and M. tuberculosis.
\end{abstract}

Keys words: Spondylodiscitis, Mycobacterium tuberculosis, Staphylococcus aureus, Empirical treatment, Morocco.

\section{INTRODUCTION}

Spondylodiscitis is rare but can cause severe symptoms. It is often recognized and treated late. The diagnosis is based on indirect (clinical, serologic, radiologic) and direct (anatomopathologic, microbiological) findings. Tuberculosis is the most frequent cause in developing countries. Other causes (parasitic, common bacteria, inflammatory) remain considerable.

Morocco is an endemic country for tuberculosis infection,

*Corresponding Author. Mailing address: Military Teaching Hospital Mohammed V, Hay Riad 10000 Rabat.; Tel.: + $212642624745 . ;$ E-mail: comlanz@yahoo.fr 
with an annual incidence of 100 per 100,000 inhabitants (12), therefore spondylodiscitis are treated considering TB as the most frequent cause.

The aim of this work is to study the bacteriological features of infectious spondylodiscitis in order to guide the initial treatment which remains empirical.

\section{PATIENTS AND METHODS}

This is a retrospective study of 4 years (2006-2009) in the bacteriological Laboratory of the Rabat Mohammed V Military Teaching Hospital. The following bacteriological investigations were carried out for each case of infectious spondylodiscitis; Gram staining, culture under aerobic and anaerobic conditions, fungal culture and Mycobacterium tuberculosis research. While the following bacteriological investigations were carried out for some cases; histopathology and blood culture (BACTEC Aerobic fluometric method). Treatment of specimen was done in Microbiological Safety Cabinet. The media used for culture were: Chapman Agar, sheep blood and chocolate Agar; all incubated at $37^{\circ} \mathrm{C}$ under aerobic environment enriched with $5 \%$ carbon dioxide.

To seek anaerobic bacteria, we used Schaedler Agar and Columbia blood Agar media incubated under strict anaerobic conditions. Systematically, we also used for all the specimens Schaedler and heart-brain broths for enrichment. The incubation period was prolonged for 10 days or more. A bacterium was incriminated when it was found in the various media used for culture in accordance with direct examination. The antibiogram was made following the recommendations of the antibiogram committee of the French Society of Microbiology (16). For Koch's bacilli diagnosis, we also investigated urine and sputum. Mycobacteria cultures were done using Lowenstein Jensen and Coletsos media associated to culture on liquid MGIT-media (Benton Dickinson 960). To identify mycobacteria, we used a molecular biology technic (NASBA Genotype Mycobacteria Direct VER 4.0 HAIN Lifescience Technique)

\section{RESULTS}

\section{Epidemiological distribution}

30 samples of consecutive patients were analysed in our laboratory during the study period. Among the patients included, there were 21 men (70\%) and 9 women (30\%) with a sex-ratio of 2.33. The mean age was 49.88 (range, 23-77 years). $30 \%$ of the cases were hospitalized in neurosurgery department followed by rheumatology department in about $33.3 \%$. The lumbar column was the most involved site in 21 cases (21/30), the dorsal 7 (7/30) and cervical column 2 (2/30).

\section{Bacterial species distribution}

M. tuberculosis was found in 10 cases (10/30) including 4 cases in association with common organisms (Propionibacterium acnes, Staphylococcus aureus, Staphylococcus epidermidis, and Corynebacterium species). Apart from M. tuberculosis, B. melitensis was the only specific pathogen found. Pyogenic bacteria were isolated in 15 cases of which 12 (12/15) revealed simple organisms including Grampositive cocci in 9 cases (9/12) with 3 cases of $S$. aureus and Gram-negative bacilli in 3 cases (3/12) with 2 cases of $P$. aeruginosa. Four samples with strong suspicion of infectious spondylodiscitis were found negative. In Table 1, we summarized the results. Blood cultures realized for 16 patients were positive in 7 cases. The anatomopathologic exams carried out for 20 patients found in 6 cases epithelioid granulomata and giants cells with caseous necrosis in full concordance with TB culture. In 11 cases non specific inflammation was found, 1 case had langherhansian hystiocytosis and 2 cases were without inflammation. 
Table 1. Bacteria found analysing 26 cases of infectious spondylodiscitis

\begin{tabular}{|c|c|c|}
\hline Germs & Species & $\mathbf{N}$ \\
\hline \multirow[t]{2}{*}{ Staphylococci } & Staphylococcus aureus & 3 \\
\hline & Staphylococcus epidermidis & 2 \\
\hline \multirow[t]{4}{*}{ Streptococci } & Group A Streptococci & 1 \\
\hline & Group B Streptococci & 1 \\
\hline & Streptococcus oralis & 1 \\
\hline & Enterococcus fecalis & 1 \\
\hline Enterobacteria & Enterobacter cloacae & 2 \\
\hline \multirow[t]{3}{*}{ Non-fermentativeGram-negative bacilli } & Pseudomonas aeruginosa & 2 \\
\hline & Acinetobacter baumanii & 1 \\
\hline & Stenotrophomonas maltophilia & 1 \\
\hline Gram-positive bacilli & Propionibacterium acnes & 1 \\
\hline \multirow[t]{2}{*}{ Specific bacteria } & Mycobacterium tuberculosis & 10 \\
\hline & Brucella militensis & 1 \\
\hline \multirow[t]{7}{*}{ Association } & S. aureus and E. faecalis & 1 \\
\hline & A. baumanii and Streptocoque & 1 \\
\hline & E. cloacae and S. maltophilia & 1 \\
\hline & M. tuberculosis and P. acnes & 1 \\
\hline & M.tuberculosis and S.epidermidis & 1 \\
\hline & M.tuberculosis and S. aureus & 1 \\
\hline & M.tuberculosis and corynebacterium sp & 1 \\
\hline
\end{tabular}

\section{DISCUSSION}

Infectious spondylodiscitis are relatively scarce diseases $(13,18)$. We involved 30 patients during the study period (4 years). Studies already published on the subject in North Africa or Europe included similar sample sizes or more $(1,2,9)$.

Our data is in line with those found in literature when we consider the prevalence of males, the high mean age of patients and the predominance of the lumbar site involvement (1, 2, 4, $5,8,9,11,13)$.

Infectious spondylodiscitis is an emergency; therefore early diagnosis and adequate antibiotherapy are required. A mortality rate of $11 \%$ is associated to spondylodiscitis and delay in diagnosis is an important risk factor (6).

Identifying the germ incriminated and its sensitivity to antibiotics are imperative especially as non TB causes are on the increase, sometimes attaining $50 \%$ of the causes (15).
In our context where TB is endemic and technical means of diagnosis of non TB causes of spondylodiscitis are lacking, many cases of spondylodiscitis are diagnosed as TB considering only epidemiological criteria.

In our study, we found the prevalence of TB causes at $33.3 \%$, with monomicrobial cultures in 6 cases (6/10). We also found Koch's bacilli in sputum and urine samples. These findings differ from those of other regions endemic for TB where specific germs (Koch's bacillus, brucella) are the predominant causes. However it is important to note that in those studies, presumptive diagnosis were included. So Ben Fredj et al. (2) found in Tunis Koch's bacillus prevalence at $39.62 \%$ but only $20.75 \%$ were well diagnosed. It is recommended to start anti TB treatment when epidemiological, clinical and radiologic findings are strongly evocative of TB. Polymicrobial causes or fungal infections must be discussed when the evolution is atypical. 
In general, Gram-positive cocci (75\%) particularly $S$. aureus (25\%) were the main pyogenic bacteria found. The other germs found were Gram-negative bacilli and Grampositive bacilli with one case of Brucella melitensis.

We didn't find any multiresistant bacteria strain. The predominance of $S$. Aureus among Gram-positive bacteria involved in spondylodiscitis has been noted by many studies and this can be explained by the bone tropism of $S$. aureus.

Our study highlighted the contribution of blood cultures in the diagnosis of spondylodiscitis. Blood cultures were positive in $43.75 \%$ of the cases. Our results are close to those found by Cherasse et al. (50\%) (3) and Legrand et al.(47.3\%) (13). Finding same germs in blood stream and osteo articular (bone and joints) sites is strongly in favour of the right diagnosis. It has been noticed that spondylodiscitis can reveal authentic bacteremia (13). Therefore blood culture must be systematical and if only one sample is positive, it must be considered (10).

Anatomopathologic examination is interesting in the quick diagnosis of TB and brucellosis (14). In our study, anatomopathology helped us to diagnose $30 \%$ of cases before TB culture positivity and allowed for early treatment. Anatomopathologic exams remain an important tool for spondylodiscitis diagnosis $(7,14,17)$.

In $13.3 \%$ of the cases we suspected infectious causes without finding the germ. It has been reported that in 8 to $30 \%$ of infectious spondylodiscitis, bacteriological tests are negative and this even if there are no technical mistakes in the diagnostic process (7). The negativity could be linked to germs known to grow with difficulty on culture medium such as $N$. gonorrhoeae, N. meningitidis, Listeria monocytogenes, or germs needing special diagnostic tools such as Mycoplasma and Ureaplasma urealyticum (7). In such cases, there is a difficulty with linking spondylodiscitis to an infectious cause. Therefore the choice of an antibiotherapy is complicated.

In such cases, the absence of specific clinical signs, the good response to the empirical antibiotherapy chosen and the absence of relapse after the treatment are helpful to link these spondylodiscitis to pyogenic bacteria.
The choice of antibiotics for empirical treatment is not consensual. The choice must take into consideration etiological factors (potential source of infection, host predispositions to some germs), clinical presentation, frequency of causative agents (Staphylococcus in general and S. aureus in particular, followed by $\mathrm{KB}$ ) and prior antibiotherapy completed by microbiological and pharmacological criteria specific to bone infections $(14,19)$.

\section{CONCLUSION}

In spite of progresses in the development of diagnostic techniques concerning spondylodiscitis, we still have spondylodiscitis with unknown causes. The management of the disease must be a multidisciplinary matter. If there is a lack in microbiological findings, local epidemiology must be considered. In morocco, the empirical treatment must take into account S. aureus and M. tuberculosis.

\section{REFERENCES}

1. Belghali, S.; Ben Fredj, H.; Ben Haj Slama, K.: Ben Smida, I.; Denguezli, N.; Ben Abdessalem, M.H. (2006). Les spondylodiscites infectieuses (SPDI) à germes banals: Étude comparative avec les SPDI a germes spécifiques. Rev Rhum 73, 1089-1259.

2. Ben Fredj, H.; Baccouche, K.; Hachfi, W.; Zeglaoui, H.; Khalifa, M.; Letaief, A. (2009). La spondylodiscite infectieuse (SI) au centre tunisien : à propos d'une série 106 cas. Med Mal Infect 39, S53-S55.

3. Cherasse, A.; Martin, D.; Tavernier, C.; Maillefert, J.F. (2003). Are blood cultures performed after disco-vertebral biopsy useful in patients with pyogenic infective spondylitis? Rheumatol 42, 913-914.

4. Cottle, L.; Riordan, T. (2008). Infectious spondylodiscitis. J Infection 56, 401- 412 .

5. Frikha, F.; Elloumi, H.; Kaddour, N.; Marzouk, S.; Jallouli, M.; Frigui, M. (2007). Les spondylodiscites infectieuses dans un service de médecine interne : 14 cas. Rev Rhum. 74, 1039-1208.

6. Genevay, S. (2006). Spondylodiscite infectieuse : le regard du rhumatologue. Rev Med Suisse 57, 715-20.

7. Gillard, J.; Boutoille, D.; Varin, S.; Asseray, N.; Berthelot, J.M.; Maugars, Y. (2005). Suspected disk space infection with negative microbiological tests-report of eight cases and comparison with documented pyogenic discitis. Joint Bone Spine 72, 156-62. 
8. Kapsalaki, E.; Gatselis, N.; Stefos, A.; Makaritsis, K.; Vassiou, A.; Fezoulidis, I. (2009). Spontaneous spondylodiscitis: presentation, risk factors, diagnosis, management, and outcome. Int J Infect Dis 13, 564-9.

9. Karadimas, E.J.; Bunger, C.; Lindblad, B.E.; Hansen, E.S.; Hoy, K.; Helmig, P. (2008). Spondylodiscitis. A retrospective study of 163 patients. Acta Orthop. 79, 650-9.

10. Khatib, R.; Schaffer, C.; Johnson, L.B. (2002). Staphylococcus aureus in a single positive blood culture: causes and outcome. Scand J Infect Dis. 34, 645-7.

11. Kochbati, S.; Ben Chihaoui, M.; Boussema, F.; Ketari, S.; Zouaoui, W.; Jemni, C. (2006). Les caractéristiques des spondylodiscites infectieuses. Rev Rhum. 73, 1089-1259.

12. Laboratoire National de Référence de la Tuberculose. (2008). Situation de tuberculose au Maroc. Rapport.

13. Legrand, E.; Flipo, R.M.; Guggenbuhl, P.; Masson, C.; Maillefert, J.F.; Soubrier, M. (2001). Management of nontuberculous infectious discitis. Treatments used in 110 patients admitted to 12 teaching hospitals in
France. Joint Bone Spine 68, 504-9.

14. Legrand, E.; Massin, P.; Levasseur, R.; Hoppé, E.; Chappard, D.; Audran, M. (2006). Stratégie diagnostique et principes thérapeutiques au cours des spondylodiscites infectieuses bactériennes. Rev Rhum 73, 373379.

15. Nolla, J.M.; Ariza, J.; Gomez-Vaquero C.; Fiter J.; Bermejo J.; Valverde, J. (2002). Spontaneous pyogenic vertebral osteomyelitis in nondrug users. Semin Arthritis Rheum 31, 271-8.

16. Soussy, C.J. (2008). Comité de l'Antibiogramme de la Société Française de Microbiologie. Recommandations.

17. Spilf. (2007). Spondylodiscites infectieuses primitives, et secondaires à un geste intradiscal, sans mise en place de matériel. Recommandations 2007. Med Mal Infect 37, 573-583.

18. Tali, E.T. (2004). Spinal infections. Eur J Radiol 50, 120-33.

19. Zeller, V.; Desplaces, N. (2006). Antibiothérapie des infections ostéoarticulaires à pyogènes chez l'adulte : principes et modalités. Rev Rhum. 73, 183-190. 